\title{
COSMIC RAYS FROM THE KNEE TO THE SECOND KNEE: $10^{14}$ TO $10^{18} \mathrm{eV}^{*}$
}

\author{
JÖRG R. HÖRANDEL \\ University of Karlsruhe, Institute for Experimental Nuclear Physics, P.O. 3640 \\ 76021 Karlsruhe, Germany - www-ik.fzk.de/ joerg
}

\begin{abstract}
The energies of cosmic rays, fully ionized charged nuclei, extend over a wide range up to $10^{20} \mathrm{eV}$. A particularly interesting energy region spans from $10^{14}$ to $10^{18} \mathrm{eV}$, where the all-particle energy spectrum exhibits two interesting structures, the 'knee' and the 'second knee'. An explanation of these features is thought to be an important step in understanding of the origin of the highenergy particles. Recent results of air shower experiments in this region are discussed. Special attention is drawn to explain the principle of air shower measurements - a simple Heitler model of (hadronic) air showers is developed.
\end{abstract}

Keywords: cosmic rays, knee, air showers, Heitler model

\section{Introduction}

The energy spectrum of cosmic rays (fully ionized atomic nuclei) spans a wide range in energy from $\mathrm{GeV}$ energies up to $10^{20} \mathrm{eV}$. Over these 10 decades the flux decreases by about 30 orders of magnitude rather featureless, following roughly a power law $d N / d E \propto E^{\gamma}$. The power law behavior indicates a non-thermal origin of the particles. To reveal small structures in the shape of the energy spectrum the flux is usually multiplied by the energy to some power. The energy spectrum multiplied by $E^{3}$ is depicted in Fig. 1. In this representation the spectrum looks rather flat and fine structures can be recognized, indicating small changes in the spectral index $\gamma$. The most important are the knee at $E_{k} \approx 4.5 \mathrm{PeV}$ where the power law spectral index changes from $\gamma=-2.7$ at low energies to $\gamma \approx-3.1$, the second knee at $E_{2 n d} \approx 400 \mathrm{PeV} \approx 92 \times E_{k}$, where the spectrum exhibits a second steepening to $\gamma \approx-3.3$, and the ankle at about $4 \mathrm{EeV}$, above

* Lecture given at the International School of Cosmic Ray Astrophysics, 15th Course: Astrophysics at Ultra-high Energies, 20-27 June 2006, Ettore Majorana Centre Erice, Sicily, Italy 


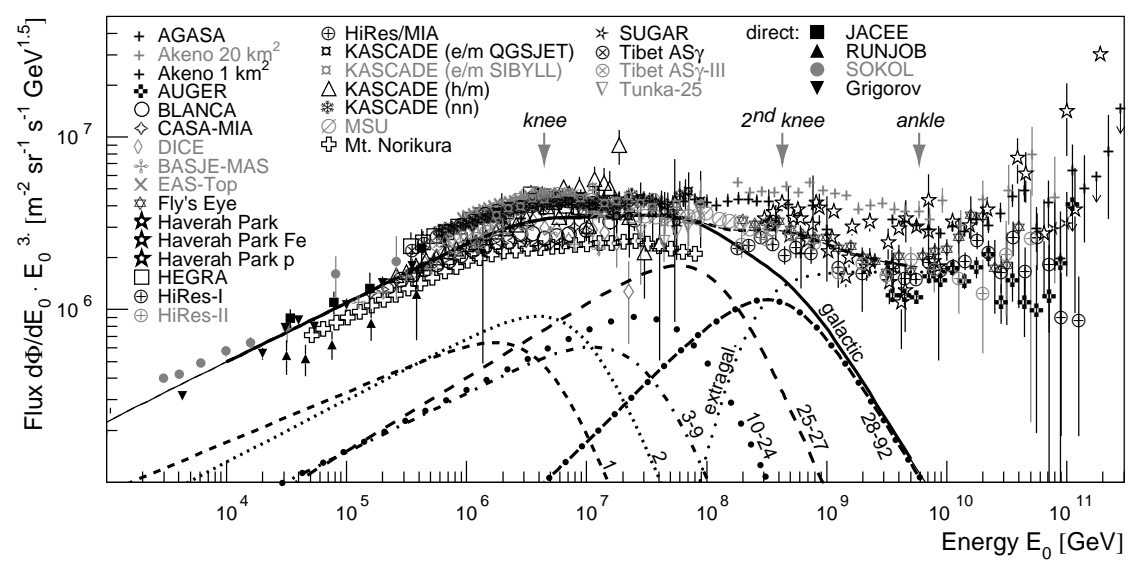

Fig. 1. All-particle energy spectrum of cosmic rays, the flux is multiplied by $E^{3}$, for references see [1]. The lines represent spectra for elemental groups (with nuclear charge numbers $Z$ as indicated) according to the poly-gonato model [2]. The sum of all elements (galactic) and a presumably extragalactic component are shown as well.

this energy the spectrum seems to flatten again to $\gamma \approx-2.7$. To understand the origin of these structures is expected to be a key element in the understanding of the origin of cosmic rays (CRs).

The lecture starts with a short overview on the physics of galactic cosmic rays (Sect. 2). Measurements in the energy region of interest are performed with air shower experiments, their principles are outlined in Sect. 3 with a simple Heitler model. Finally, recent results of air shower experiments are reviewed in Sect. 4.

\section{Galactic Cosmic Rays and the Knee}

\subsection{Sources}

At energies around $1 \mathrm{GeV} / \mathrm{n}$ all elements known from the periodic table with nuclear charge number $Z$ from 1 to 92 have been found in CRs [2-4]. Overall, the abundance of elements in CRs is very similar to the abundance found in the solar system, which indicates that CRs are "regular matter" but accelerated to very high energies. This is emphasized by measurements of the CRIS experiment [5] which show that the abundances of particular isotopes in cosmic rays and in the solar system differ by less than $20 \%$.

The bulk of CRs is assumed to be accelerated in blast waves of supernova remnants (SNRs). This goes back to an idea by Baade and Zwicky who proposed SNRs as cosmic-rays sources due to energy balance considerations 
[6]. They realized that the power necessary to sustain the cosmic-ray flux could be provided when a small fraction $\sim 10 \%$ of the kinetical energy released in supernova explosions is converted into CRs. Fermi proposed a mechanism to accelerate particles with moving magnetic clouds [7]. This led to todays picture that the particles are accelerated at strong shock fronts in SNRs through first-order Fermi acceleration [8-12]. This theory predicts spectra at the sources following a power law $d N / d E \propto E^{-2.1}$.

Diffusive, first-order shock acceleration works by virtue of the fact that particles gain an amount of energy $\Delta E \propto E$ at each cycle, when a cycle consists of a particle passing from the upstream (unshocked) region to the downstream region and back. At each cycle, there is a probability that the particle is lost downstream and does not return to the shock. Higher energy particles are those that remain longer in the vicinity of the shock and have enough time to achieve the high energy. After a time $T$ the maximum energy attained is $E_{\max } \sim Z e \beta_{s} B T V_{s}$, where $\beta_{s}=V_{s} / c$ is the velocity of the shock. This results in an upper limit, assuming a minimal diffusion length equal to the Larmor radius of a particle of charge $Z e$ in the magnetic fields $B$ behind and ahead of the shock. Using typical values of Type II supernovae exploding in an average interstellar medium yields $E_{\max } \approx Z \cdot 100 \mathrm{TeV}$ [13]. More recent estimates give a maximum energy up to one order of magnitude larger for some types of supernovae $E_{\max } \approx Z \cdot 5 \mathrm{PeV}$ [14-16]. As the maximum energy depends on the charge $Z$, heavier nuclei (with larger $Z$ ) can be accelerated to higher energies. This leads to consecutive cut-offs of the energy spectra for individual elements proportional to their charge $Z$, starting with the proton component.

This theory is strongly supported by recent measurements of the HESS experiment $[17,18]$, observing $\mathrm{TeV} \gamma$-rays from the shell type SNR RX J1713.7-3946. For the first time, a SNR could be spatially resolved in $\gamma$ rays and spectra have been derived directly at a potential cosmic-ray source. The measurements yield a spectral index $\gamma=-2.19 \pm 0.09 \pm 0.15$ for the observed $\gamma$-ray flux. The results are compatible with a nonlinear kinetic theory of cosmic-ray acceleration in supernova remnants and imply that this supernova remnant is an effective source of nuclear CRs, where about $10 \%$ of the mechanical explosion energy are converted into nuclear CRs [19].

\subsection{Propagation}

After acceleration, the particles propagate in a diffusive process through the Galaxy, being deflected many times by the randomly oriented magnetic fields $(B \approx 3 \mu \mathrm{G})$. The nuclei are not confined to the galactic disc, they 
propagate in the galactic halo as well. The diffuse $\gamma$-ray background, extending well above the disc, detected by the EGRET experiment, exhibits a structure in the $\mathrm{GeV}$ region, which is interpreted as indication for the interaction of propagating CRs with interstellar matter [20]. The $\gamma$-rays are produced in inelastic hadronic reactions of CRs with the interstellar medium (ISM) via neutral pion decay $p+\operatorname{ISM} \rightarrow \pi^{0} \rightarrow \gamma \gamma$. The height of the propagation region in the halo has been estimated measuring the ${ }^{10} \mathrm{Be} /{ }^{9} \mathrm{Be}-$ ratio with the ISOMAX experiment to be a few kpc [21]. Determining the abundance of radioactive nuclei, which decay on the way from the source to the Earth, allows to determine the residence time of CRs in the Galaxy. Measurements with the CRIS instrument yield about $15 \cdot 10^{6}$ a for particles with $\mathrm{GeV}$ energies [22].

Information on the propagation pathlength of CRs is often derived from the measurement of the ratio of primary to secondary nuclei. The latter are produced through spallation during propagation in the Galaxy. The energy dependence of the measured ratio is frequently explained in Leaky Box models by a decreasing pathlength of CRs in the Galaxy $\Lambda(R)=$ $\Lambda_{0}\left(R / R_{0}\right)^{-\delta}$, with typical values $\Lambda_{0} \approx 10-15 \mathrm{~g} / \mathrm{cm}^{2}, \delta \approx 0.5-0.6$, and the rigidity $R_{0} \approx 4 \mathrm{GV}$ [23]. In this picture the spectra observed at Earth should be steeper as compared to the source, i.e. the spectral index $\gamma$ should be smaller by the value of $\delta$.

Energy spectra of individual elements have been measured up to energies of about $10^{14} \mathrm{eV}$ by experiments above the atmosphere, the results being well compatible with power laws $[2,4]$. Due to spallation during the propagation process, the spectra of heavy elements are slightly flatter as compared to light nuclei $[2,24]$, e.g. comparing protons $\gamma_{p}=-2.71 \pm 0.02$ to iron nuclei $\gamma_{F e}=-2.59 \pm 0.06$.

The regular component of the galactic magnetic field will cause particles with charge $Z$ to describe helical trajectories with a Larmor radius $R_{L}=$ $p /\left(Z e B_{0}\right)=1.08 \mathrm{pc} \cdot E[\mathrm{PeV}] /\left(Z \cdot B_{0}[\mu \mathrm{G}]\right)$, while the random field component causes diffusive propagation. With increasing energy (or momentum) it becomes more difficult to magnetically confine the particles to the Galaxy. Since $R_{L} \propto 1 / Z$ it is expected that leakage from the Galaxy occurs for light elements (low $Z$ ) earlier as compared to heavy nuclei, i.e. protons leak first and subsequently all other elements start to escape from the Galaxy.

\subsection{Structures in the Energy Spectrum}

Many possible origins for the knee are discussed in the literature $[25,26]$. Most popular are assumptions of a finite energy attained during the accel- 
eration process and leakage from the Galaxy as discussed. In both scenarios the energy spectra of elements exhibit a cut-off at an energy proportional to the nuclear charge $Z$ and the knee in the all-particle spectrum is caused by the cut-off of protons.

All other elements follow subsequently and above a certain energy no more particles are left. On the other hand, the measured all-particle flux extends up to $10^{20} \mathrm{eV}$, and the highest-energy particles are usually being considered of extragalactic origin. The Larmor radius of a proton with an energy of $10^{20} \mathrm{eV}$ in the galactic magnetic field is $R_{L} \approx 36 \mathrm{kpc}$, comparable to the diameter of the Galaxy. This emphasizes that such high-energy particles are of extragalactic origin. The transition region from galactic to extragalactic CRs is of particular interest, key features are the origin of the second knee and the ankle.

Reviewing the properties of CRs accelerated in SNRs, Hillas finds that a second (galactic) component is necessary to explain the observed flux at energies above $10^{16} \mathrm{eV}$ [27]. Another possibility is a significant contribution of ultra-heavy elements (heavier than iron) to the all-particle flux at energies around $400 \mathrm{PeV}[2,24]$. In this approach the second knee is caused by the fall-off of the heaviest elements with $Z$ up to 92 . It is remarkable that the second knee occurs at $E_{2 n d} \approx 92 \times E_{k}$, the latter being the energy of the first knee. The dip seen in the spectrum between $10^{18}$ and $10^{19} \mathrm{eV}$, see Fig. 1, is proposed to be caused by electron-positron pair production of CRs on cosmic microwave background photons [28] $p+\gamma_{3 K} \rightarrow p+e^{+}+e^{-}$.

\section{Measurement Techniques}

To clarify the situation and to distinguish between the different models, measurements of the flux of individual elements, or at least groups of elements, up to high energies are necessary. Direct measurements above the atmosphere on stratospheric balloons up to energies exceeding $10^{14} \mathrm{eV}$ are performed with various instruments like ATIC [29], CREAM [30], BESS [31], or TRACER [32]. The presently largest experiment with singleelement resolution, TRACER, has an aperture of $5 \mathrm{~m}^{2} \mathrm{sr}$. With an exposure of $50 \mathrm{~m}^{2} \mathrm{sr}$ d accumulated during a circumpolar flight in 2003 energy spectra could be measured up to about $5 \cdot 10^{14} \mathrm{eV}$ for oxygen and $8 \cdot 10^{13} \mathrm{eV}$ for iron nuclei [33].

To extend the measurements to energies beyond the knee, at present, ground based installations are the only possibility. With these experiments, secondary products generated in the atmosphere are measured, the extensive air showers (EAS). Air showers were discovered in 1938 by W. 
Kolhörster [34] and independently by P. Auger [35]. Auger describes his work in a book [36] translated by the director of this school M.M. Shapiro.

In todays experiments, the energy is basically derived from the number of particles observed and the primary's mass is estimated by measurements of the depth of the shower maximum (Sect. 3.1.5) or the electron-to-muonratio (Sect. 3.1.6). Two types of experiments may be distinguished: installations measuring the longitudinal development of showers in the atmosphere and apparatus measuring the density (and energy) of secondary particles at ground level.

An example for the latter is the KASCADE experiment [37], covering an area of $200 \times 200 \mathrm{~m}^{2}$. The basic idea is to measure the electromagnetic component in an array of unshielded scintillation detectors and the muons in scintillation counters shielded by a lead and iron absorber, while the hadronic component is measured in a large calorimeter [38]. The total number of particles at observation level is obtained through the measurement of particle densities and the integration of the lateral density distribution [39]. The direction of air showers is reconstructed through the measurement of the arrival time of the shower particles in the individual detectors.

The depth of the shower maximum is measured in two ways. Lightintegrating Cerenkov detectors like the BLANCA [40] or TUNKA [41] experiments are in principle arrays of photomultiplier tubes with light collection cones looking upwards in the night sky, measuring the lateral distribution of Cerenkov light at ground level. The depth of the shower maximum and the shower energy is derived from these observations. Imaging telescopes as in the HiRes [42] or AUGER [43] experiments observe an image of the shower on the sky through measurement of fluorescence light, emitted by nitrogen molecules, which had been excited by air shower particles.

\subsection{A Heitler Model for Air Showers}

The basic properties of EAS are illustrated using a Heitler model [44], expanding an approach by Matthews [45]. The principle ideas of the model are emphasized by full EAS simulations using the CORSIKA code [46] with the hadronic interaction models FLUKA [47] and QGSJET 01 [48]. For the latter, a modification with lower cross-sections has been used [49]. ${ }^{a}$

\footnotetext{
aertical showers with fixed energies between $10^{5}$ and $3.16 \cdot 10^{10} \mathrm{GeV}$ in steps of half a decade have been calculated. Thresholds for photons, electrons, muons, and hadrons were chosen as $E_{\gamma}>0.25 \mathrm{MeV}, E_{e}>0.25 \mathrm{MeV}, E_{\mu}>100 \mathrm{MeV}$, and $E_{h}>100 \mathrm{MeV}$.
} 

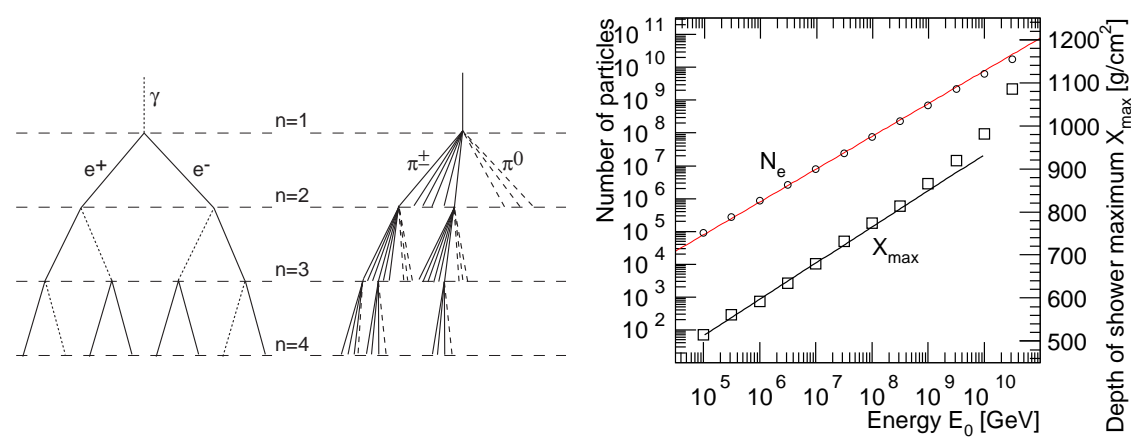

Fig. 2. Left: Schematic view of an electromagnetic cascade (left) and a hadronic shower (right). Not all pion lines are shown [45]. Right: Number of electrons at shower maximum and depth of the shower maximum as function of photon energy. The lines are according to (1) and (2).

\subsubsection{Electromagnetic Cascades}

A simple approximation of an electromagnetic cascade is shown schematically in Fig. 2. A primary photon generates an $e^{+} e^{-}$pair. An electron radiates a single photon after traveling one splitting length $d=X_{0} \ln 2$, where $X_{0}$ is the radiation length $\left(X_{0}^{a i r}=36.66 \mathrm{~g} / \mathrm{cm}^{2}\right)$. An electron looses on average half of its energy through radiation over the distance $d$. After traveling the same distance a photon splits into an $e^{+} e^{-}$pair. In either instance, the energy of a particle is assumed to be equally divided between two outgoing particles. After $n$ splitting lengths, at a distance $x=n X_{0} \ln 2$, the total shower size (electrons and photons) is $N=2^{n}=\exp \left(x / X_{0}\right)$ and the initial energy $E_{0}$ is distributed over $N$ particles. The splitting continues until the energy per particle $E_{0} / N$ is too low for pair production or bremsstrahlung. Heitler takes this energy to be the critical energy $\left(E_{c}^{e}=85 \mathrm{MeV}\right.$ in air), at which ionization losses and radiative losses are equal.

A shower initiated by a primary photon reaches its maximum size $N_{\max }$ when all particles have the energy $E_{c}^{e}$, which means $E_{0}=E_{c}^{e} N_{\max }$. The penetration depth $X_{\max }$ at which the shower reaches its maximum is obtained by determining the number $n_{c}$ of splitting lengths, required to reduce the energy per particle to $E_{c}^{e}$. Since $N_{\max }=2^{n_{c}}$, the number of splitting lengths is $n_{c}=\ln \left(E_{0} / E_{c}^{e}\right) / \ln 2$, giving $N_{\max }=E_{0} / E_{c}^{e}$ and

$$
X_{\text {max }}^{\gamma}=n_{c} X_{0} \ln 2=X_{0} \ln \left(E_{0} / E_{c}^{e}\right) .
$$

The elongation rate $\Lambda$ specifies the increase of $X_{\max }$ with energy $E_{0}$ and is defined as $\Lambda \equiv \mathrm{d} X_{\max } / \mathrm{d} \lg E_{0}$. Using (1) gives $\Lambda^{\gamma}=\ln 10 X_{0}=84.4 \mathrm{~g} / \mathrm{cm}^{2}$ 
per decade of primary energy for electromagnetic showers in air. Thus, $X_{\max }=597 \mathrm{~g} / \mathrm{cm}^{2}+84 \mathrm{~g} / \mathrm{cm}^{2} \lg \left(E_{0} / \mathrm{PeV}\right)$ is expected. This prediction agrees well with results of full simulations as can be inferred from Fig. 2. ${ }^{\mathrm{b}}$

The simple model describes quite well the position of the maximum of electromagnetic cascades when compared to EAS simulations and to measurements at accelerators $[45,52]$. However, the model overestimates the actual ratio of electrons to photons. It predicts that after a few generations the electron size approaches $N_{e} \approx \frac{2}{3} N_{\max }$. This is much too large for several reasons, mainly that multiple photons are often radiated during bremsstrahlung and many electrons and positrons range out in the air.

To extract the number of electrons $N_{e}^{\max }$ at shower maximum from Heitler's total size $N_{\max }$, a simple correction

$$
N_{e}^{\max }=\frac{E_{0}}{g E_{c}^{e}}
$$

is adopted, with a constant value $g$. When the estimated electron number is compared to measurements, the factor $g$ has to be fine tuned. It depends on properties of the detectors used like the energy threshold and the efficiency to detect photons and electrons (or positrons). Comparisons with results at accelerators indicate values between $g=10$ [45] and $g=20$ [52]. Results of a full EAS simulation are depicted in Fig. 2. For electromagnetic showers the number of electrons turns out to be almost exactly linearly proportional to the shower energy as expected from (2). A fit yields $N_{e} \propto E_{0}^{0.97}$ and a correction factor $g \approx 13$ is obtained, compatible with the accelerator based results. With this value the number of electrons at shower maximum is according to $(2) N_{e}^{\max }=9.0 \cdot 10^{5} \cdot E_{0} / \mathrm{PeV}$.

\subsubsection{Hadronic Showers}

Hadron induced showers are modeled using a similar approach, for a figurative sketch, see Fig. 2. The atmosphere is divided in layers of fixed thickness $\lambda_{i} \ln 2$, where $\lambda_{i}$ is the interaction length of strongly interacting particles. An energy around $100 \mathrm{GeV}$ is a typical energy for pions in air showers and for a simple approach a constant value $\lambda_{i}=120 \mathrm{~g} / \mathrm{cm}^{2}$ is adopted. Hadrons interact after traversing one layer, producing $N_{c h}$ charged pions and $\frac{1}{2} N_{c h}$ neutral pions. The latter decay promptly to photons, initiating electromagnetic cascades. Charged pions continue through another layer and interact.

${ }^{\mathrm{b}}$ The deviations at high energies are due to the Landau Pomeranchuk Migdal effect $[50,51]$. 
The process continues until the charged pions fall bellow the critical energy $E_{c}^{\pi}$, where they are all assumed to decay, yielding muons.

The multiplicity of charged particles produced in hadron interactions increases very slowly with laboratory energy $\propto E^{0.2}$ in $p p$ and $p \bar{p}$ data [53]. The multiplicity in $\pi-{ }^{14} \mathrm{~N}$ collisions increases as $N_{c h} \approx 5,11$, and 27 at 10 , 100 , and $10^{4} \mathrm{GeV}$, respectively [49]. A constant value $N_{c h}=10$ is adopted in the following for the number of charged particles produced in pion-air interactions, again corresponding to an energy of about $100 \mathrm{GeV}$.

The second parameter is the energy $E_{c}^{\pi}$ at which further particle production by $\pi^{ \pm}$ceases. $E_{c}^{\pi}$ may be defined as the energy at which the probability for decay and hadronic interaction equalize. Following Ref. [45] a constant critical pion energy $E_{c}^{\pi}=20 \mathrm{GeV}$ is adopted in the following.

If we consider a proton with $E_{0}$ entering the atmosphere, we have after $n$ interactions $N_{\pi}=\left(N_{c h}\right)^{n}$ charged pions. Assuming equal division of energy during particle production, these pions carry a total energy of $(2 / 3)^{n} E_{0}$. The remainder of the energy goes into electromagnetic showers from $\pi^{0}$ decays. Hence, the energy per charged pion is $E_{\pi}=E_{0} /\left(\frac{3}{2} N_{c h}\right)^{n}$. After a certain number $n_{c}$ of generations, $E_{\pi}$ becomes less than $E_{c}^{\pi}$. The number of interactions needed to reach $E_{\pi}=E_{c}^{\pi}$ is

$$
n_{c}=\frac{\ln E_{0} / E_{c}^{\pi}}{\ln \frac{3}{2} N_{c h}}=0.85 \lg \left(\frac{E_{0}}{E_{c}^{\pi}}\right) \text {. }
$$

\subsubsection{Number of Muons}

The number of muons is obtained, assuming that all pions decay, using $N_{\mu}=N_{\pi}=\left(N_{c h}\right)^{n_{c}}$. Their energy dependence is derived applying (3)

$$
\ln N_{\mu}=n_{c} \ln N_{c h}=\beta \ln \left(\frac{E_{0}}{E_{c}^{\pi}}\right), \quad \text { with } \quad \beta=\frac{\ln N_{c h}}{\ln \frac{3}{2} N_{c h}} \approx 0.85
$$

for $N_{c h}=10$. It should be noted that although $N_{c h}$ changes (slowly) as the shower develops, $\beta$ depends only logarithmically on this value.

So far, an important aspect of hadronic interactions has been neglected. In an interaction only a fraction of the energy is available for secondary particle production, usually characterized by the the inelasticity $\kappa$. Taking this effect into account, in an interaction initiated by a particle with energy $E$, the energy $(1-\kappa) E$ is taken away by a single leading particle, $\frac{2}{3} \kappa E$ is used to produce $N_{c h}$ charged pions, and $\frac{1}{3} \kappa E$ goes via neutral pions into the electromagnetic component. Including inelasticity in the Heitler model 
changes the parameter $\beta$ in (4) to [45]

$$
\beta=\frac{\ln \left[1+N_{c h}\right]}{\ln \left[\left(1+N_{c h}\right) /\left(1-\frac{1}{3} \kappa\right)\right]} \approx 1-\frac{\kappa}{3 \ln \left(N_{c h}\right)}=1-0.14 \kappa .
$$

The elasticity for the most energetic meson in pion-air interactions yields $1-\kappa$ between 0.26 and 0.32 [49], resulting in $\beta=0.90$.

To expand the simple approach from primary protons to nuclei, the superposition model is used. A nucleus with atomic mass number $A$ and energy $E_{0}$ is taken to be $A$ individual single nucleons, each with energy $E_{0} / A$, and each acting independently. The resulting EAS is treated as the sum of $A$ individual proton induced showers, all starting at the same point. The observable shower features are obtained by substituting the lower primary energy into the expressions derived for proton showers and summing $A$ such showers. Applying this to the number of muons yields $N_{\mu}=A\left(E_{0} /\left(A E_{c}^{\pi}\right)\right)^{\beta}$. The number of muons in showers induced by nuclei with mass number $A$ and energy $E_{0}$ is then

$$
N_{\mu}=\left(\frac{E_{0}}{E_{c}^{\pi}}\right)^{\beta} A^{1-\beta} \approx 1.69 \cdot 10^{4} \cdot A^{0.10}\left(\frac{E_{0}}{1 \mathrm{PeV}}\right)^{0.90} .
$$

Two important features follow from (6): the number of muons increases as function of energy slightly less than exactly linear and $N_{\mu}$ increases as function of the mass of the primary particle as $\propto A^{0.1}$. Accordingly, iron induced showers contain about 1.5 times as many muons as proton showers with the same energy. This results from the less-than-linear growth of the number of muons with energy $-\beta<1$ in (6). The lower energy nucleons which initiate the shower generate fewer interaction generations, and consequently, loose less energy to the electromagnetic component.

The number of muons at shower maximum as function of energy is shown in Fig. 3 as obtained from full simulations. The lines indicate predictions according to (6), being well in agreement with the simulations.

\subsubsection{Number of Electrons}

Conservation of energy implies that the primary energy is split into electromagnetic and hadronic parts $E_{0}=E_{e m}+E_{h}$. The number of electrons is estimated using this relation. The hadronic energy appears in the simple approach in the muon component as $E_{h}=N_{\mu} E_{c}^{\pi}$ and the energy fraction for the electromagnetic component is, using (6)

$$
\frac{E_{e m}}{E_{0}}=\frac{E_{0}-N_{\mu} E_{c}^{\pi}}{E_{0}}=1-\left(\frac{E_{0}}{A E_{c}^{\pi}}\right)^{\beta-1} \text {. }
$$



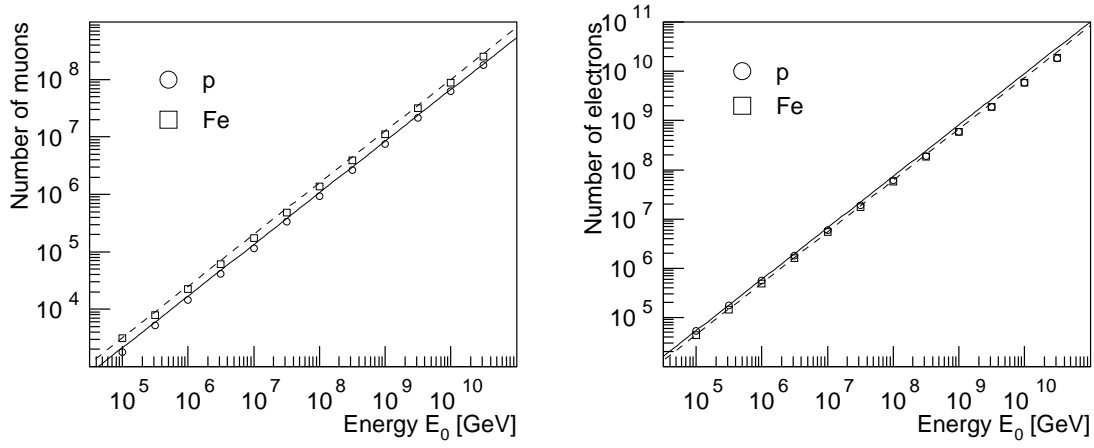

Fig. 3. Number of muons (left) and number of electrons (right) at shower maximum as function of energy for primary protons and iron nuclei according to CORSIKA simulations (symbols). The lines are predictions according to (6) and (10), respectively, for protons (-) and iron (---) nuclei.

The electromagnetic fraction is $57 \%$ at $E_{0}=10^{14} \mathrm{eV}$, increasing to $79 \%$ at $10^{17} \mathrm{eV}$ for proton induced showers. For iron induced showers the fraction rises from $38 \%$ to $68 \%$.

Equation (7) can be approximated by a power law

$$
\frac{E_{e m}}{E_{0}} \approx a\left(\frac{E_{0}}{A E_{c}^{\pi}}\right)^{b} \text {. }
$$

Series expansion near $x_{0}=E_{0} / E_{c}^{\pi}=10^{5}$ yields the number of electrons at shower maximum as function of energy

$$
N_{e}=\frac{E_{e m}}{g E_{c}^{e}} \approx \frac{a}{g E_{c}^{e}}\left(A E_{c}^{\pi}\right)^{-b} E_{0}^{1+b}
$$

with $b=(1-\beta) /\left(x_{0}^{1-\beta}-1\right) \approx 0.046$ and $a=\left(1-x_{0}^{\beta-1}\right) /\left(x_{0}^{b}\right) \approx 0.40$. Hence, $\alpha=1+b \approx 1.046$ is obtained, which leads, using $g=13$ to

$$
N_{e} \approx 5.95 \cdot 10^{5} \cdot A^{-0.046}\left(\frac{E_{0}}{1 \mathrm{PeV}}\right)^{1.046} .
$$

This implies that the number of electrons grows as function of energy slightly faster than exactly linear. The electron number decreases with increasing mass number, an iron induced shower is expected to contain about $83 \%$ of the electromagnetic energy of a proton shower with the same energy. It should be emphasized that the model does not take into account absorption in the atmosphere, thus, the number of electrons obtained is valid at shower maximum. 
The number of electrons at shower maximum according to full simulations is shown as function of energy in Fig. 3. The results are compared to predictions according to (10) for proton and iron induced showers indicated by the lines. It can be seen that the simple model reproduces quite well the results of the full simulations.

\subsubsection{Depth of the Shower Maximum}

The atmospheric depth at which the electromagnetic shower component reaches its maximum is called $X_{\max }$. In hadronic interactions $\kappa / 3$ of the available energy goes into the electromagnetic component via $\pi^{0}$-decays, see Fig. 2. For a simple estimate only the first generation of electromagnetic showers is used. This approach will certainly underestimate the value of $X_{\max }$ since it neglects the following subshowers.

The first interaction occurs at an atmospheric depth $X_{1}=\lambda_{i} \ln 2$, where $\lambda_{i}$ is the interaction length of a primary proton $\lambda_{i}^{p-a i r}$. The latter can be approximated around $1 \mathrm{PeV}$ by the relation

$$
\lambda_{i}^{p-a i r}=\xi+\zeta \lg \frac{E_{0}}{1 \mathrm{PeV}}
$$

with $\xi=68.55 \mathrm{~g} / \mathrm{cm}^{2}$ and $\zeta=-4.88 \mathrm{~g} / \mathrm{cm}^{2}$.

In the first interaction $\frac{1}{2} N_{c h}$ neutral pions are produced, yielding $N_{c h}$ photons. Each photon initiates an electromagnetic cascade with the energy $\kappa E_{0} /\left(3 N_{c h}\right)$, developing in parallel with the others. The average multiplicity of charged particles produced in pion-nitrogen interactions [49] can be parameterized for energies around $1 \mathrm{PeV}$ as

$$
N_{c h}=N_{0}\left(\frac{E_{0}}{1 \mathrm{PeV}}\right)^{\eta}
$$

with $N_{0}=55.2$ and $\eta=0.13$.

The depth of the shower maximum is obtained as in (1) for an electromagnetic shower with an energy $\kappa E_{0} /\left(3 N_{c h}\right)$, starting after the first interaction at a depth $X_{1}, X_{\max }^{p}=\lambda_{i}^{p-a i r} \ln 2+X_{0} \ln \left(\kappa E_{0} /\left(3 N_{c h} E_{c}^{e}\right)\right)$. Using (11) and (12), the expression

$$
X_{\text {max }}^{p}=\xi \ln 2-X_{0} \ln \left(\frac{3 N_{0}}{\kappa} \frac{E_{c}^{e}}{\mathrm{PeV}}\right)+\left(X_{0} \ln 10-\eta X_{0} \ln 10+\zeta \ln 2\right) \lg \frac{E_{0}}{\mathrm{PeV}}
$$

is obtained. The elongation rate for protons is determined by the elongation rate for electromagnetic showers $\Lambda^{\gamma}=X_{0} \ln 10$ and in addition by terms which take into account the growing multiplicity of secondary particles, as 


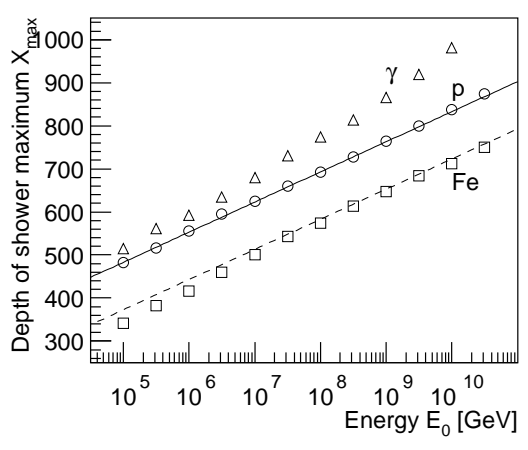

well as the decreasing interaction length as function of energy. Taking the numerical parameters as described, the elongation rate $\Lambda^{p}=(84.4-11.0-$ $3.4) \mathrm{g} / \mathrm{cm}^{2}=70.0 \mathrm{~g} / \mathrm{cm}^{2}$ is obtained, and one realizes that the effect of growing multiplicity dominates the effect of a decreasing interaction length by about a factor three. Evaluating also the constant term in (13) yields $X_{\max }^{p}=442.9 \mathrm{~g} / \mathrm{cm}^{2}+70.0 \mathrm{~g} / \mathrm{cm}^{2} \lg \left(E_{0} / \mathrm{PeV}\right)$.

When compared to results of full simulations, the calculated values for $X_{\max }^{p}$ are about $110 \mathrm{~g} / \mathrm{cm}^{2}$ or almost $2 \lambda_{i}^{p-a i r}$ smaller than the results of full calculations depicted in Fig. 4 (dashed line). Presumably this is a consequence of neglecting the contributions of following generations of $\pi^{0}$ production. However, the predicted elongation rate agrees extremely well with the value obtained from the CORSIKA simulations at $1 \mathrm{PeV}$ $\Lambda^{p}=69.9 \pm 0.3 \mathrm{~g} / \mathrm{cm}^{2}$ per decade. The solid line represents (13) shifted upwards by $110 \mathrm{~g} / \mathrm{cm}^{2}$ and agrees well with the proton simulations.

To expand the simple approach from primary protons to nuclei with mass number $A$, the superposition model is used and in (13) the energy $E_{0}$ is substituted by $E_{0} / A$. This yields $X_{\max }^{A}=X_{\max }^{p}-X_{0} \ln A$, predicting that the maximum for iron induced showers should be about $150 \mathrm{~g} / \mathrm{cm}^{2}$ higher up in the atmosphere. In the full simulations, the difference is slightly smaller as can be inferred from the figure.

\subsubsection{Energy and Mass of the Primary Particle}

In EAS experiments the reconstructed number of electrons and muons are often presented in the $\lg N_{\mu}-\lg N_{e}$ plane in order to estimate the energy and mass of the shower inducing particles. As an application of the simple Heitler Model, lines of constant mass and energy in the $N_{\mu}-N_{e}$ plane are derived in the following to illustrate the method utilized in the experiments. 

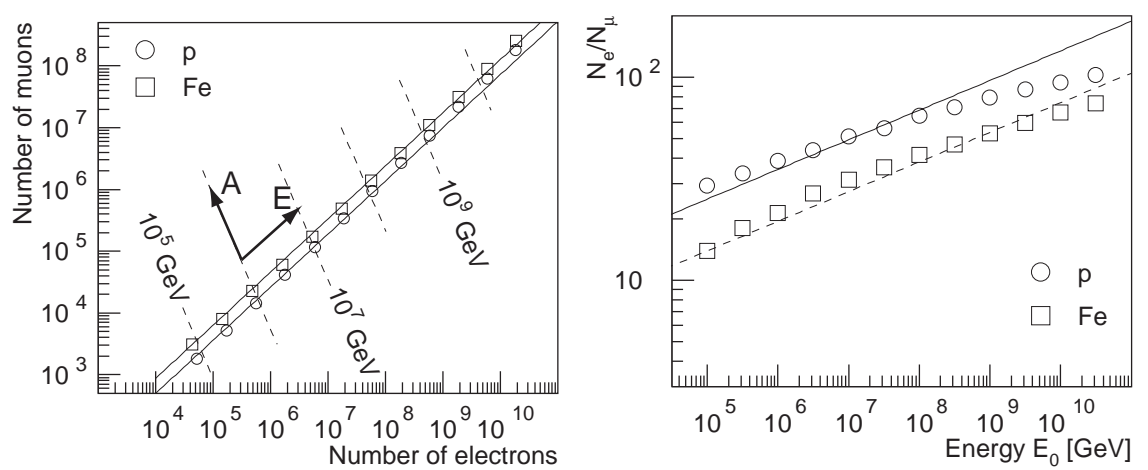

Fig. 5. Left: Average number of muons versus number of electrons at shower maximum for primary protons and iron nuclei. The data points are results of full simulations. The solid lines represent (14) for protons and iron nuclei, the dashed lines are equal energy lines according to (15). Right: Ratio of electron to muon number $N_{e} / N_{\mu}$ at shower maximum as function of energy for primary protons and iron nuclei. The data points are results of full simulations. The lines indicate (16) for the two primaries.

To deduce lines of constant mass, $(9)$ is transformed to obtain $E_{0}$, which, in turn is introduced into (6). This yields the number of muons as function of the number of electrons at shower maximum

$$
\left.N_{\mu}\right|_{A}=\left(E_{c}^{\pi}\right)^{-\delta}\left(\frac{g E_{c}^{e}}{a}\right)^{\delta} A^{1-\delta} N_{e}^{\delta} \approx 0.18 \cdot A^{0.14} N_{e}^{0.86}
$$

with the exponent $\delta=\beta /(1+b) \approx 0.86$. In a similar way, lines of constant energy are derived. $A$ is taken from (9) and put in (6), which leads to

$$
\left.N_{\mu}\right|_{E_{0}}=\frac{1}{E_{c}^{\pi}}\left(\frac{g E_{c}^{e}}{a}\right)^{\varepsilon} E_{0}^{\beta+\varepsilon(b-1)} N_{e}^{\varepsilon} \approx 5.77 \cdot 10^{16}\left(\frac{E_{0}}{1 \mathrm{PeV}}\right)^{2.97} N_{e}^{-2.17}
$$

with an exponent $\varepsilon=-(1-\beta) / b \approx-2.17$.

The constant-mass lines for protons and iron nuclei are shown in Fig. 5 together with equal-energy lines for energies from $10^{5}$ to $10^{10} \mathrm{GeV}$. These sets of lines form a coordinate system for energy and mass in the $N_{\mu}-N_{e}$ plane. The axis are non-perpendicular to each other. In the figure also results of full CORSIKA simulations for proton and iron induced showers are shown for fixed energies from $10^{5}$ to $3.16 \cdot 10^{10} \mathrm{GeV}$ in steps of half a decade. Taking the simplicity of the model into account the predicted lines agree quite well with the full simulations and they give a good illustration of the physics in the $N_{\mu}-N_{e}$ plane.

Dividing (9) by (6) yields the electron-to-muon ratio at shower maxi- 
mum

$$
\frac{N_{e}}{N_{\mu}}=\frac{a}{g E_{c}^{e}}\left(E_{c}^{\pi}\right)^{\beta-b}\left(\frac{E_{0}}{A}\right)^{1+b-\beta} \approx 35.1 \cdot\left(\frac{E_{0}}{1 \mathrm{PeV} \cdot A}\right)^{0.15}
$$

It depends on the energy per nucleon $E_{0} / A$ of the primary particle. This is the reason why the ratio $N_{e} / N_{\mu}$ is frequently used in EAS experiments to estimate the mass of the primary particle. If the energy is derived from another observable, the mass can be inferred. Predictions according to (16) are compared to results of full simulations for proton and iron induced showers in Fig. 5. The simple model predicts the calculated ratio quite well. The CORSIKA simulations exhibit almost a power law behavior, however, at high energies some flattening with respect to the predicted slope is visible.

\section{Experimental Results}

The all-particle energy spectra obtained by many experiments are compiled in Fig. 1. Shown are results from direct measurements above the atmosphere as well as from various air shower experiments. The individual measurements agree within a factor of two or three in the flux and a similar shape can be recognized for all experiments with a knee at energies of about $4 \mathrm{PeV}$. Typical values for the systematic uncertainties of the absolute energy scale for air shower experiments are about 15 to $20 \%$. Renormalizing the energy scales of the individual experiments to match the all-particle spectrum obtained by direct measurements in the energy region up to almost a PeV requires correction factors of the order of $\pm 10 \%$ [2]. Indicating that the all-particle spectrum seems to be well determined.

Due to the large fluctuations in air showers it is not possible to derive energy spectra for individual elements from air shower data. Therefore, frequently the mean mass of CRs is investigated. An often-used quantity to characterize the composition is the mean logarithmic mass, defined as $\langle\ln A\rangle=\sum_{i} r_{i} \ln A_{i}, r_{i}$ being the relative fraction of nuclei of mass $A_{i}$. Investigating the ratio of the number of electrons and muons at ground level and the average depth of the shower maximum an increase of the mean logarithmic mass in the energy range around the knee could be observed by many experiments [1]. Such an increase is expected from consecutive cut-offs of the energy spectra of individual elements, starting with protons.

A significant step forward in understanding the origin of CRs are measurements of energy spectra for individual elements or at least groups of elements. Up to about a $\mathrm{PeV}$ direct measurements have been performed with instruments above the atmosphere. As examples, results for primary 

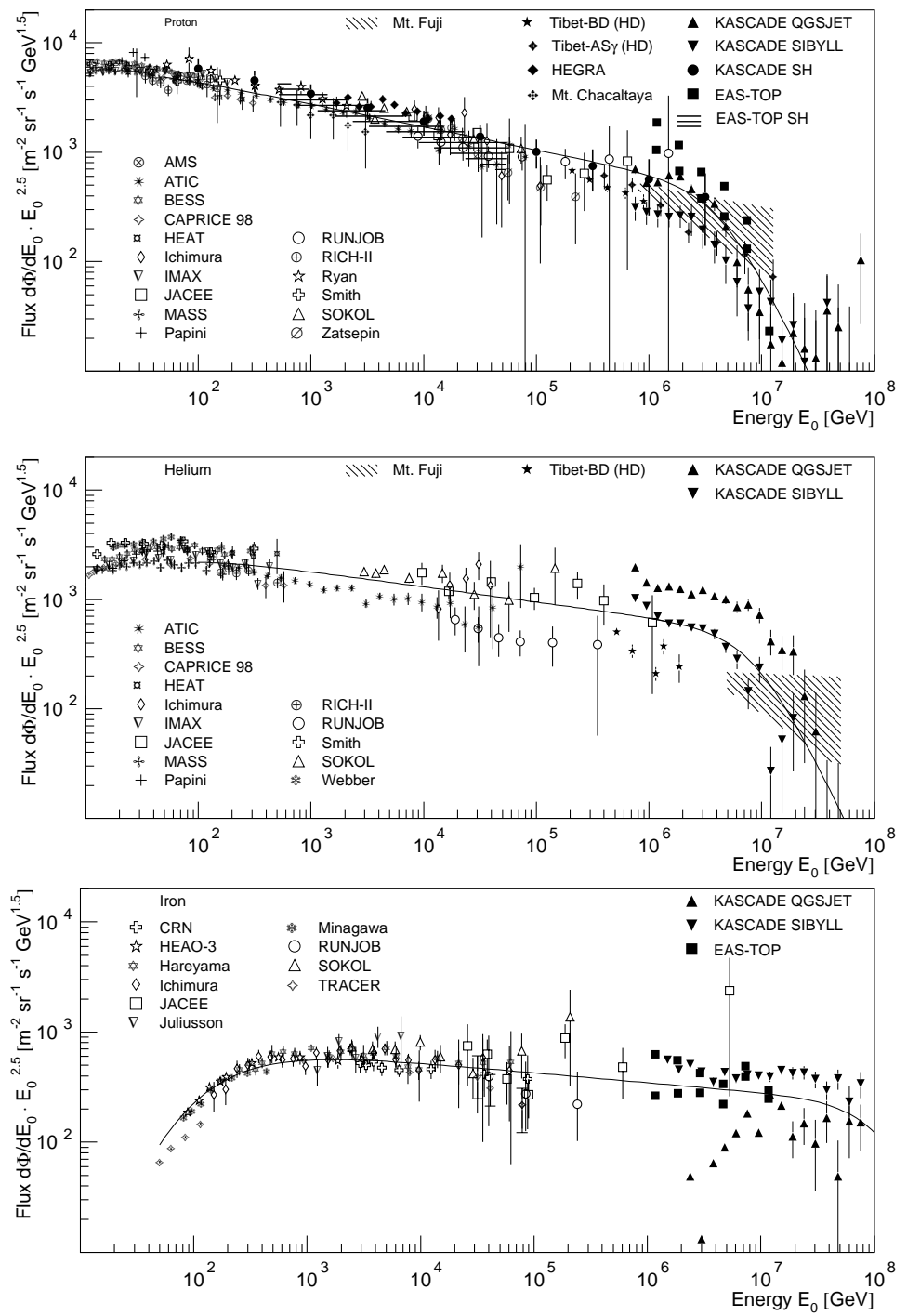

Fig. 6. Energy spectra for primary protons, helium, and iron nuclei from direct and indirect measurements for references see [1]. The lines indicate spectra according to the poly-gonato model [2].

protons, helium, and iron nuclei are compiled in Fig. 6. Recently, also indirect measurements of elemental groups became possible.

With the KASCADE experiment, the problem of composition has been 

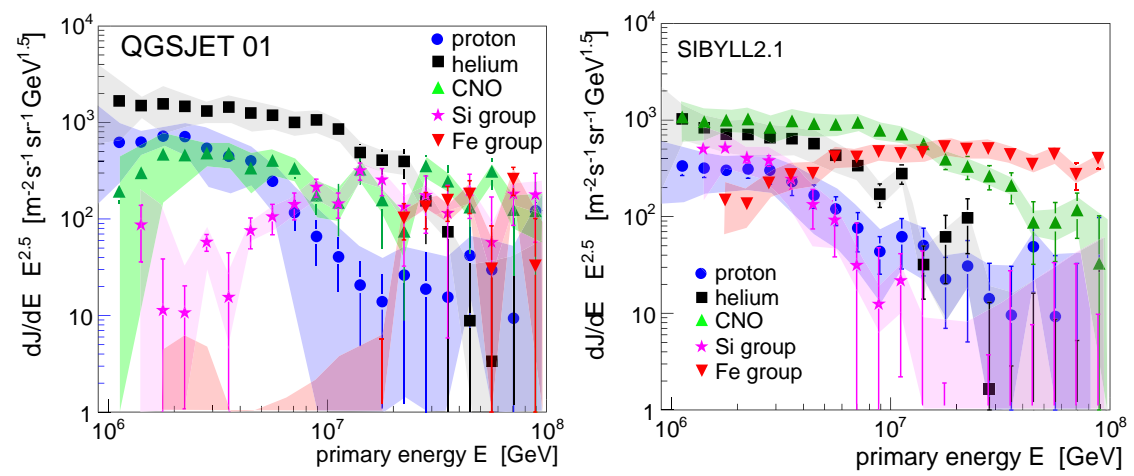

Fig. 7. Energy spectra for elemental groups as obtained by the KASCADE experiment, using two different models (QGSJET 01 and SIBYLL 2.1) to interpret hadronic interactions in the atmosphere [54].

approached in various ways [55]. An advanced analysis is founded on the measurement of the electromagnetic and muonic shower components [54]. It is based on the deconvolution of a two-dimensional electron muon number distribution. Unfolding is performed using two hadronic interaction models (QGSJET 01 and SIBYLL 2.1) to interpret the data. The spectra obtained for five elemental groups are displayed in Fig. 7. They exhibit sequential cut-offs in the flux for the light elements. For both models a depression is visible for protons around 3 to $4 \mathrm{PeV}$ and at higher energies for helium nuclei. The systematic differences in flux for the spectra derived with QGSJET and SIBYLL amount to a factor of about two to three. The silicon and iron groups show a rather unexpected behavior for both models. The increase of the flux for both groups (QGSJET) and the early cut-off for the silicon group (SIBYLL) is not compatible with contemporary astrophysical models. The discrepancies are attributed to the fact that none of the air shower models is able to describe the observed data set in the whole energy range consistently [54].

The KASCADE results are compared to results of other experiments in Fig. 6. EAS-TOP derived spectra from the simultaneous observation of the electromagnetic and muonic components. HEGRA used an imaging Čerenkov telescope system to derive the primary proton flux [56]. The primary proton flux has been derived from measurements of the flux of unaccompanied hadrons at ground level with the EAS-TOP and KASCADE experiments $[57,58]$. Spectra for protons and helium nuclei are obtained from emulsion chambers exposed at Mts. Fuji and Kanbala [59]. The Tibet group performs measurements with a burst detector as well as with 
emulsion chambers and an air shower array $[60,61]$.

Considering the energy range above $10 \mathrm{GeV}$, at least a qualitative picture of the energy spectra for individual mass groups emerges: the spectra seem to be compatible with power laws with a cut-off at high energies. The spectra according to the poly gonato model [2] are indicated in the figures as lines. It can be recognized that the measured values are compatible with cut-offs at energies proportional to the nuclear charge $\hat{E}_{Z}=Z \cdot 4.5 \mathrm{PeV}$. The lines in Fig. 1 indicate spectra for the same model. Summing up the flux of all elements, the all-particle flux is compatible with the flux derived from air shower experiments in the knee region. Above $10^{8} \mathrm{GeV}$ the flux of galactic CRs is not sufficient to account for the observed all-particle spectrum, and an additional, presumably extragalactic component is required.

Energy spectra have been reconstructed with KASCADE data up to energies of $100 \mathrm{PeV}$. At these energies statistical errors start to dominate the overall error. To improve this situation, the experiment has been enlarged. Covering an area of $0.5 \mathrm{~km}^{2}, 37$ detector stations, containing $10 \mathrm{~m}^{2}$ of plastic scintillators each, have been installed to extend the original KASCADE set-up [62]. Regular measurements with this new array and the original KASCADE detectors, forming the KASCADE-Grande experiment, are performed since summer 2003 [63]. The objective is to reconstruct energy spectra for groups of elements up to $10^{18} \mathrm{eV}$ [64], covering the energy region of the second knee, where the galactic cosmic ray spectrum is expected to end [65]. First analyses extend the lateral distributions of electrons and muons up to $600 \mathrm{~m}[66,67]$. Based on one year of measurements, already energies close to $10^{18} \mathrm{eV}$ are reached. It is planned to conduct an unfolding analysis, similar to the one described above, and reveal the energy spectra for groups of elements up to $10^{18} \mathrm{eV}$.

A more detailed discussion of experimental results may be found elsewhere $[1,26,68,69]$.

\section{Conclusion and Outlook}

In the last decade the understanding of the origin of high-energy CRs has advanced significantly. In particular, the KASCADE experiment has shown that the origin of the knee in the all-particle energy spectrum is due to a cut-off of the light elements. A corresponding increase of the mean mass as function of energy in the knee region is observed by many experiments. Such a behavior is expected from astrophysical models, explaining the knee due to a finite energy reached in the acceleration process and due to leakage from the Galaxy. However, it has also evolved that the astrophysical inter- 
pretation of air shower data, at present, is limited by the understanding of high-energy hadronic interactions in the atmosphere. Experiments like KASCADE have reached the sensitivity to improve interaction models and corresponding analyses are under way, e.g. [70-72]. A big step forward is the observation of $\mathrm{TeV}-\gamma$-rays from SNRs with the expected spectral index $\gamma \approx-2.1$, thus giving an important hint to the sources of hadronic CRs.

In the next years the KASCADE-Grande experiment and the Ice Cube/Ice Top experiment at the south pole [73] will measure CRs in the energy region of the second knee and will provide information on the mass composition in this region, where the galactic CR component is expected to end. Balloon borne experiments like ATIC, CREAM, or TRACER will improve the knowledge about CR propagation, by extending the energy spectra of individual elements to energies approaching the knee.

\section{Acknowledgment}

It was a pleasure to participate in an inspiring school and to experience the great hospitality in Erice. I would like to thank Jim Matthews for our exchange about the Heitler model and acknowledge the stimulating scientific discussions with my colleagues from the KASCADE-Grande, TRACER, and AUGER experiments.

\section{References}

1. J. Hörandel, J. Phys.: Conf. Ser. 47 (2006) 41.

2. J. Hörandel, Astropart. Phys. 19 (2003) 193.

3. M. Shapiro, R. Silberberg, Ann. Rev. Nucl. Sci. 20 (1970) 323.

4. B. Wiebel-Soth et al., Astron. \& Astroph. 330 (1998) 389.

5. M. Wiedenbeck et al., 28th Int. Cosmic Ray Conf., Tsukuba 4 (2003) 1899.

6. W. Baade, F. Zwicky, Phys. Rev. 46 (1934) 76.

7. E. Fermi, Phys. Rev. 75 (1949) 1169.

8. W. Axford et al., 15th Int. Cosmic Ray Conf., Plovdiv 11 (1977) 132.

9. G. Krymsky, Dok. Acad. Nauk USSR 234 (1977) 1306.

10. A. Bell, Mon. Not. R. Astr. Soc. 182 (1978) 147.

11. R. Blanford, J. Ostriker, Astrophys. J. 221 (1978) L29.

12. M. Longair, High Energy Astrophysics vol. 2, Cambridge, 1994.

13. P. Lagage, C. Cesarsky, Astron. \& Astroph. 125 (1983) 249.

14. E. Berezhko, Astropart. Phys. 5 (1996) 367.

15. K. Kobayakawa et al., Phys. Rev. D 66 (2002) 083004.

16. L. Sveshnikova et al., Astron. \& Astroph. 409 (2003) 799

17. F. Aharonian et al., Nature 432 (2004) 75.

18. F. Aharonian et al., Astron. \& Astroph. 449 (2006) 223.

19. H. Völk, E. Berezhko, Astron. \& Astroph. 451 (2006) 981. 
20. A. Strong, I. Moskalenko, preprint astro-ph/9903370 (1999).

21. A. Molnar, M. Simon, 28th Int. Cosmic Ray Conf., Tsukuba 4 (2003) 1937.

22. N. Yanasak et al., Astrophys. J. 563 (2001) 768.

23. S. Stephens, R. Streitmatter, Astrophys. J. 505 (1998) 266.

24. J. Hörandel et al., astro-ph/0609490 (2006).

25. J. Hörandel, Astropart. Phys. 21 (2004) 241.

26. J. Hörandel, Int. J. Mod. Phys. A 20 (2005) 6753.

27. A. Hillas, J. Phys. G: Nucl. Part. Phys. 31 (2005) R95.

28. V. Berezinsky, astro-ph/0509069 (2005).

29. T. Guzik et al., Adv. Space Res. (2004) in press.

30. E. Seo et al., Adv. Space Res. 33 (2004) 1777.

31. Y. Ajima et al., Nucl. Instr. \& Meth. A 443 (2000) 71.

32. F. Gahbauer et al., Astrophys. J. 607 (2004) 333.

33. D. Müller et al., 29th Int. Cosmic Ray Conf., Pune 3 (2005) 89.

34. W. Kolhörster et al., Naturw. 26 (1938) 576.

35. P. Auger et al., Comptes renduz 206 (1938) 1721.

36. P. Auger, What are Cosmic Rays, University of Chicago Press, 1945.

37. T. Antoni et al., Nucl. Instr. \& Meth. A 513 (2003) 490.

38. J. Engler et al., Nucl. Instr. \& Meth. A 427 (1999) 528.

39. T. Antoni et al., Astropart. Phys. 14 (2001) 245.

40. J. Fowler et al., Astropart. Phys. 15 (2001) 49.

41. O. Gress et al., Nucl. Phys. B (Proc. Suppl.) 75A (1999) 299.

42. T. Abu-Zayyad et al., Astrophys. J. 557 (2000) 686.

43. J. Abraham et al., Nucl. Instr. \& Meth. A 523 (2004) 50.

44. W. Heitler, The quantum theory of radiation, Oxford, 1954.

45. J. Matthews, Astropart. Phys. 22 (2005) 387.

46. D. Heck et al., Report FZKA 6019, Forschungszentrum Karlsruhe (1998).

47. A. Fassò et al., FLUKA: Status and Prospective of Hadronic Applications, Proc. Monte Carlo 2000 Conf., Lisbon, A. Kling, F. Barao, M. Nakagawa, P. Vaz eds., Springer (Berlin), 2001, p. 955.

48. N. Kalmykov et al., Nucl. Phys. B (Proc. Suppl.) 52B (1997) 17.

49. J. Hörandel, J. Phys. G: Nucl. Part. Phys. 29 (2003) 2439.

50. L. Landau, I. Pomeranchuk, Dokl. Akad. Nauk SSSR 92 (1953) 535 and 735.

51. A. Migdal, Phys. Rev. 103 (1956) 1811.

52. S. Plewnia et al., Nucl. Instr. \& Meth. A 566 (2006) 422.

53. S. Eidelman et al., Phys. Lett. B 592 (2004) 1.

54. T. Antoni et al., Astropart. Phys. 24 (2005) 1.

55. J. Hörandel et al., J. Phys.: Conf. Ser. 39 (2006) 463.

56. F. Aharonian et al., Phys. Rev. D 59 (1999) 092003.

57. M. Aglietta et al., Astropart. Phys. 19 (2003) 329.

58. T. Antoni et al., Astrophys. J. 612 (2004) 914.

59. J. Huang et al., Astropart. Phys. 18 (2003) 637.

60. M. Amenomori et al., Phys. Rev. D 62 (2000) 112002.

61. M. Amenomori et al., 28th Int. Cosmic Ray Conf., Tsukuba 1 (2003) 143.

62. G. Navarra et al., Nucl. Instr. \& Meth. A 518 (2004) 207.

63. A. Chiavassa et al., 29th Int. Cosmic Ray Conf., Pune 6 (2005) 313. 
64. A. Haungs et al., J. Phys.: Conf. Ser. 47 (2006) 238.

65. J. Hörandel et al., J. Phys.: Conf. Ser. 47 (2006) 132.

66. R. Glasstetter et al., 29th Int. Cosmic Ray Conf., Pune 6 (2005) 293.

67. J. v. Buren et al., 29th Int. Cosmic Ray Conf., Pune 6 (2005) 301.

68. S. Swordy et al., Astropart. Phys. 18 (2002) 129.

69. J. Hörandel, Neutrinos and Explosive Events in the Universe, M.M. Shapiro, T. Stanev, J.P. Wefel (eds.) NATO Science Series, Springer, 2005, p. 365.

70. J. Hörandel et al., Nucl. Phys. B (Proc. Suppl.) 151 (2006) 205.

71. J. Hörandel et al., 29th Int. Cosmic Ray Conf., Pune 6 (2005) 121.

72. J. Milke et al., 29th Int. Cosmic Ray Conf., Pune 6 (2005) 125.

73. T. Gaisser et al., 28th Int. Cosmic Ray Conf., Tsukuba 2 (2003) 1117. 